\title{
Laboratory Experiment of Blind Adaptive Array with Subcarrier Transmission Power Assignment in Spectrum Superposing Scenarios ${ }^{\dagger}$
}

\author{
Hideya So ${ }^{1}$, Kazuki Maruta ${ }^{2, *, \ddagger(D)}$ and Kouhei Suzaki ${ }^{1}$ \\ 1 NTT Network Innovation Laboratories, 1-1, Hikarinooka, Yokosuka-shi, Kanagawa 239-0847, Japan; \\ sou.hideya@lab.ntt.co.jp (H.S.); suzaki.kouhei@lab.ntt.co.jp (K.S.) \\ 2 NTT Access Network Service Systems Laboratories, 1-1, Hikarinooka, Yokosuka-shi, \\ Kanagawa 239-0847, Japan \\ * Correspondence: maruta@chiba-u.jp; Tel.: +81-43-290-3314 \\ + This paper is an extended version of our paper published in the IEEE 84th Vehicular Technology Conference \\ (VTC2016-Fall), Montréal, QC, Canada, 18-21 September 2016. \\ $\ddagger$ Current address: Graduate School of Engineering, Chiba University, 1-33 Yayoi-cho, Inage-ku, \\ Chiba-shi 263-8522, Japan.
}

Received: 14 November 2017; Accepted: 9 January 2018; Published: 11 January 2018

\begin{abstract}
This paper experimentally validates the basic feasibility of our proposed blind adaptive array (BAA) with subcarrier transmission power assignment (STPA) scheme using a prototype. The proposed STPA-BAA enables spectrum superposing without any channel state information between two different wireless communication systems: employing STPA-BAA to the secondary system, inter-system interference from/to the primary system can be suppressed. Exploiting the characteristics of constant modulus algorithm (CMA) and power inversion (PI), the secondary transmitter provides two levels of power density for each subcarrier: high and low levels. It enables the secondary receiver to suppress interference with almost the same level of the desired signal. It is also effective in reducing interference to the primary receiver that has no interference suppression function. This paper conducts laboratory experiments of spectrum superposition in two multicarrier systems by wired setup. Though the practical performance of STPA-BAA is limited due to the quantization level or dynamic range of the transceiver, effectiveness of the proposed scheme is confirmed.
\end{abstract}

Keywords: adaptive array; blind algorithm; subcarrier transmit power assignment; spectrum superposing; field programmable gate array

\section{Introduction}

Since many kinds of wireless communication systems such as wireless fidelity (Wi-Fi), worldwide interoperability for microwave access (WiMAX) or long-term evolution (LTE) (-Advanced) have emerged, frequency resources are becoming exhausted, especially in the microwave band. In order to accommodate the explosive increase in data traffic, many approaches are now being actively discussed, e.g., the use of higher frequencies, small cell deployment, and Multiple-Input Multiple-Output (MIMO) enhancement [1,2]. All of them are essential in improving the spectral efficiency. To further enhance frequency resource utilization, the spectrum sharing approach, well known as cognitive radio [3], is being investigated. The basic principle is opportunistic spectrum access (OSA) or dynamic spectrum access (DSA) [4,5]: the secondary system detects which time/frequency resources are active or which are idle, and instantly accesses vacant channels so as not to interfere with the primary systems. It can maximize the use of available frequency resources, but spectral utilization 
efficiency cannot be increased beyond 100\%. This approach also necessitates spectrum usage of the primary system. The secondary system should perform request-to-send (RTS) and clear-to-send (CTS) handshake, rendezvous protocols [6,7] or requires signaling knowledge of the primary transmitter [5]. These preprocessing might cause non-negligible overhead. A concept for utilizing unlicensed spectrum such as LTE in unlicensed spectrum (LTE-U) [8] or licensed assisted access (LAA) [9] has emerged as one of the most promising solutions to the spectrum exhaustion. Although unlicensed spectrum can be effectively utilized by the LTE function, there is apprehension that it will cause inter-system interference for Wi-Fi systems. With the LAA, listen before talk (LBT) [10] is essential to ensure co-existence with $\mathrm{Wi}-\mathrm{Fi}$, but it still permits resource sharing in time domain. Therefore, spectrum sharing in a spatial domain, i.e., spectrum superposing, is a significant approach to further enhance the spectral efficiency. Opportunistic analysis for the spectrum superposing among different wireless communication systems was conducted [11]. It derived a possibility of spectrum sharing in spatial domain for the secondary system, but its communication range is limited to be short. To expand the spectrum superposing region, some additional processing could be required. The null-steering approach $[12,13]$ disclosed the spectral efficiency improvement by suppressing inter-system interference in the superposed spectrum. The most important issue in order to realize fully spectrum superposing is how to estimate channel state information (CSI) of both primary and secondary systems since obtaining such information about different systems (inter-system CSI) is impossible. Given the above constraint, the following problems must be resolved:

- suppressing inter-system interference from the primary transmitter at the secondary receiver;

- reducing inter-system interference to the primary receiver by the secondary transmitter;

- both interference suppression/reduction must be realized in a blind manner.

Our original approach is to exploit the blind adaptive array (BAA) signal processing. It is quite effective in suppressing interference without inter-system CSI estimation. Constant modulus algorithm (CMA) $[14,15]$ and power inversion (PI) $[16,17]$ are well known BAA algorithms that do not require any a priori information such as a reference signal or direction of arrival. However, these algorithms are limited to some operational signal-to-interference power ratio (SIR) range at the input of receiver antenna elements. CMA cannot suppress the interference when SIR is smaller than $0 \mathrm{~dB}$ and PI cannot suppress it when SIR is greater than $0 \mathrm{~dB}$. In other words, these algorithms face strong limits on their applicable region when the desired signal and the interference signal have almost the same power $(\mathrm{SIR} \simeq 0 \mathrm{~dB})$. We then proposed a subcarrier transmission power assignment (STPA) [18,19], wherein the transmitter provides two levels of power density for each subcarrier: high and low levels. The receiver then applies CMA to high-level subcarriers and PI to low-level subcarriers so that these algorithms precisely work to suppress interference. It is regarded that the proposed STPA-BAA exploits power spectral densities as a priori information that can be implicitly shared between the transmitter and the receiver. The secondary receiver can successfully suppress the inter-system interference from the primary transmitter when SIR is equal to about $0 \mathrm{~dB}$. Furthermore, STPA can improve an operational SIR for the primary system since low-level subcarriers of the secondary transmitter decreases the interference power to the primary receiver. Additionally, forward error correction (FEC) functions of the primary receiver also can compensate the strong interference influenced by high-level subcarriers. The fundamental effectiveness of the proposal was clarified through computer simulations under the scenario where two orthogonal frequency division multiplexing (OFDM) systems share the superposed spectrum [19]. Our approach can realize spectral efficiency improvement without any a priori information or sensing mechanisms. Its comprehensive comparison is summarized in Table 1. However, the practical performances of STPA-BAA appeared to be limited due to the hardware specifications, e.g., quantization or dynamic range of the transceiver. To elucidate this concern, the effectiveness of the STPA-BAA proposal was reported by fabricating and testing a prototype based on filter bank multicarrier transmission systems [20]. A major contribution of this paper is to expand its significance in terms of the detailed hardware implementation and results. 
The rest of this paper is organized as follows. Section 2 describes the system model and our previously proposed STPA-BAA scheme. Sections 3 and 4 present prototype specification and experiment results, respectively. Finally, Section 5 concludes the paper.

Table 1. Comparisons in spectrum sharing/superposing approaches. DSA: dynamic spectrum access, CSI: channel state information.

\begin{tabular}{cccc}
\hline & DSA & Null-Steering & Proposed \\
\hline Spectral efficiency & $\leq 100 \%$ & $\geq 100 \%$ & $\geq 100 \%$ \\
Sharing domain & Time/Frequency & Space & Space \\
Requirement & Spectrum usage of primary system & Inter-system CSI & - \\
\hline
\end{tabular}

\section{System Model and Proposed Scheme}

\subsection{System Model}

Figure 1 shows the system model considered in this paper. Assuming a multicarrier transmission system, the secondary system with STPA-BAA is laid over the primary one on the superpose spectrum. It is noted that the primary system does not have the interference suppression function. Basically, in the cognitive radio network, the secondary system must not interfere with the primary system on the same frequency resources. Therefore, the secondary system should suppress interference from the primary transmitter as well as reduce interference to the primary receiver. $\operatorname{SIR}_{n}$ and $\operatorname{SIR}_{\text {total }}$ are defined as the SIR at the $n$-th $(1 \leq n \leq N)$ subcarrier and the total SIR among all subcarriers, respectively. Their relationship can be formulated as

$$
\begin{aligned}
\operatorname{SIR}_{n}= & \frac{\mathrm{S}_{n}}{\mathrm{I}_{n},} \\
\mathrm{SIR}_{\text {total }}= & \frac{\sum_{n=1}^{N} \mathrm{~S}_{n}}{\sum_{n=1}^{N} \mathrm{I}_{n}},
\end{aligned}
$$

where $S_{n}$ and $I_{n}$ stand for the desired and interference signal power at the $n$-th subcarrier, respectively. Let $x_{l}(n, i)$ denote the $i$-th symbol on the $n$-th subcarrier in the signal received by the $l$-th $(1 \leq l \leq L)$ antenna element. The $L$-by- 1 received signal vector of the secondary system, $\mathbf{X}(n, i)$, is defined as

$$
\mathbf{X}(n, i)=\left[\begin{array}{llll}
x_{1}(n, i) & x_{2}(n, i) & \cdots & x_{L}(n, i),
\end{array}\right]^{T},
$$

where $(.)^{T}$ denotes transposition. Received signal includes the desired signal for the secondary system and the inter-system interference signal from the primary one. The inter-system interference can be suppressed by BAA, which multiplies the received signal per subcarrier by a weight vector.

\subsection{Proposal: Blind Adaptive Array with Subcarrier Transmission Power Assignment}

Conventional BAA algorithms such as CMA and PI cannot suppress the interference enough when $\operatorname{SIR}_{\text {total }}$ is equal to $0 \mathrm{~dB}$ [18]. Focusing on multicarrier systems, the secondary transmitter assigns different power densities to each subcarrier, that is, higher and lower level. At the secondary receiver, CMA and PI are selectively applied according to assigned power densities. 


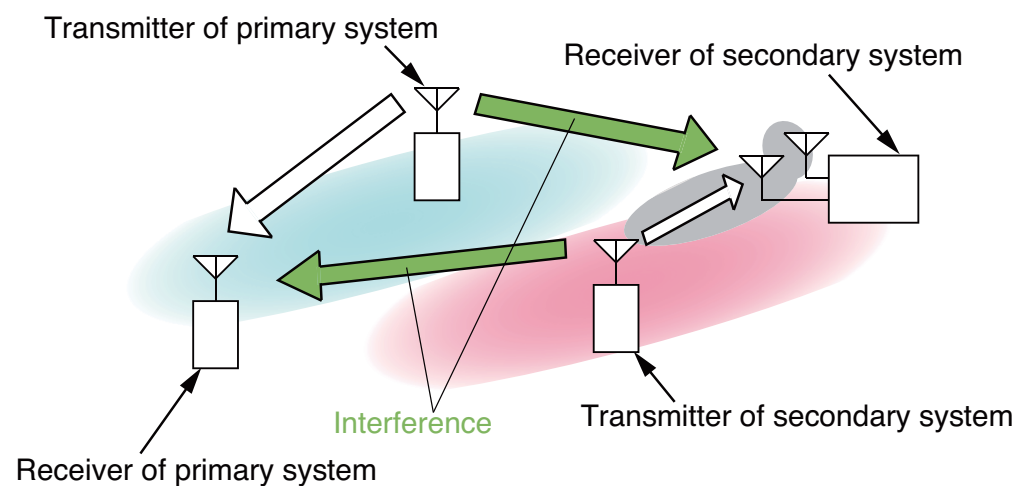

Figure 1. System model.

The concept of the proposed scheme is shown in Figure 2. $G$ is defined as the power density difference between high and low-level subcarriers. $N_{\mathrm{High}}$ is also defined as the number of high-level subcarriers. The pattern of one high-level subcarrier and multiple low-level subcarriers is assumed to be repeatedly arranged and total transmission power is controlled so as to equal that of the conventional one. When the interference from the primary system, transmitted at equal power spectral density, reaches the receiver of the secondary system, we can obtain the situation that $\operatorname{SIR}_{n}$ of high-level subcarriers exceeds $0 \mathrm{~dB}$ while that of low-level subcarriers drops below $0 \mathrm{~dB}$. The secondary system applies CMA to high-level subcarriers and PI to low-level subcarriers. This approach enables the interference suppression when $\mathrm{SIR}_{\text {total }}$ is almost equal to $0 \mathrm{~dB}$. Detailed algorithms of CMA and PI follow.

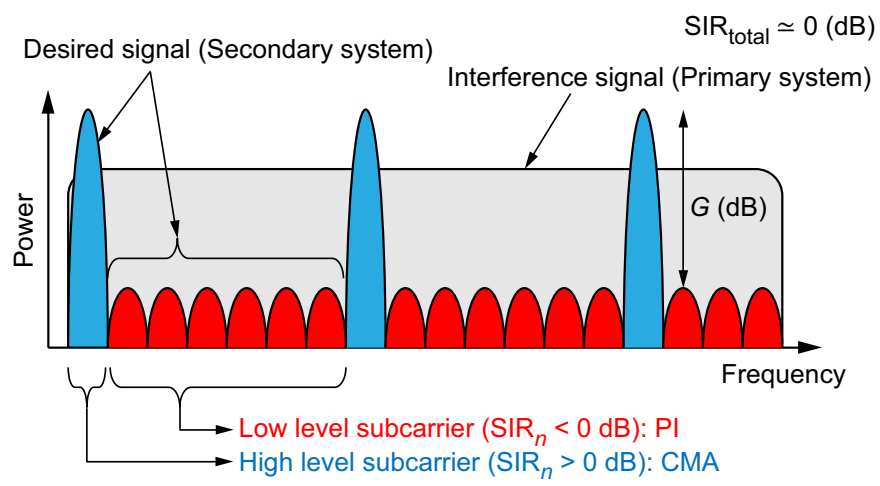

Figure 2. Concept of blind adaptive array with subcarrier transmission power assignment (STPA-BAA). SIR: signal-to-interference power ratio; CMA: constant modulus algorithm; PI: power inversion.

\subsubsection{Constant Modulus Algorithm (CMA)}

$\mathbf{W}_{\mathrm{CMA}}(n)$ defined as the $L$-by- 1 CMA weight vector at the $n$-th subcarrier. CMA adapts the array output signal, $y(n, i)$, in order to yield a constant envelope by minimizing the following cost function:

$$
\begin{aligned}
Q\left(\mathbf{W}_{\mathrm{CMA}}(n)\right) & =\mathrm{E}\left[\left.|| y(n, i)\right|^{p}-\left.\sigma^{p}\right|^{q}\right] \\
& =\mathrm{E}\left[\left.|| \mathbf{W}_{\mathrm{CMA}}^{H}(n) \mathbf{X}(n, i)\right|^{p}-\left.\sigma^{p}\right|^{q}\right],
\end{aligned}
$$

where $\mathrm{E}[$.$] and (.)^{H}$ denote ensemble mean and Hermitian transposition, respectively. $\sigma$ is a constant envelope value and generally takes the value of one. $p$ and $q$ are positive integers, and take a value of one or two, respectively. 
A number of algorithms were investigated to minimize Equation (4). We adopt least square CMA (LS-CMA) [15], which is known to exhibit superior convergence performance. CMA weight at the $m$-th iteration order, $\mathbf{W}_{\mathrm{CMA}}^{(m)}(n)$, is updated in the following manner:

$$
\begin{gathered}
\mathbf{W}_{\mathrm{CMA}}^{(m+1)}(n)=\mathbf{W}_{\mathrm{CMA}}^{(m)}(n)-\left[\sum_{i=1}^{S} \mathbf{X}(n, i) \mathbf{X}^{H}(n, i)\right]^{-1} \sum_{i=1}^{S} \mathbf{X}(n, i)\left[y^{*}(n, i)-\delta^{*}(n, i)\right] \\
=\left[\sum_{i=1}^{S} \mathbf{X}(n, i) \mathbf{X}^{H}(n, i)\right]^{-1} \sum_{i=1}^{S} \mathbf{X}(n, i) \delta^{*}(n, i), \\
\delta(n, i)=\frac{\sigma}{|y(n, i)|} y(n, i), \\
\mathbf{W}_{\mathrm{CMA}}^{(0)}(n)=\left[\begin{array}{llll}
1 & 0 & \cdots & 0
\end{array}\right]^{T},
\end{gathered}
$$

where $p=1$ and $q=2 . m(=0,1,2, \ldots), S$, and $(.)^{*}$ denote the index of CMA iteration, the index of sample, and complex conjugate, respectively. With the CMA, the desired signal must be captured at the initial array output. It indicates that the $\operatorname{SIR}_{n}$ of the initial array output must be larger than $0 \mathrm{~dB}$.

\subsubsection{Power Inversion (PI)}

PI has a property to invert the power ratio of the received signals by suppressing the signal having larger power. This leads to PI being able to effectively work when the power of the desired signal is smaller than that of the interference signal, i.e., $\mathrm{SIR}<0 \mathrm{~dB}$. The PI weight, $\mathbf{W}_{\mathrm{PI}}(n)$, is calculated by autocorrelation matrix of the received signal, $\mathbf{R}_{x x}(n)$. They are defined as

$$
\begin{aligned}
& \mathbf{W}_{\mathrm{PI}}(n)=\mathbf{R}_{x x}^{-1}(n) \mathbf{C}, \\
\mathbf{R}_{x x}(n) & =\mathrm{E}\left[\mathbf{X}(n, i) \mathbf{X}^{H}(n, i)\right] \\
& =\frac{1}{S} \sum_{i=1}^{S}\left[\mathbf{X}(n, i) \mathbf{X}^{H}(n, i)\right], \\
\mathbf{C} & =\left[\begin{array}{llll}
1 & 0 & \cdots & 0
\end{array}\right]^{T},
\end{aligned}
$$

where $\mathbf{C}$ denotes a constraint vector.

As described, PI simply inverts the power ratio of incoming signals. Its interference suppression performance is limited in smaller absolute SIR value. $\mathbf{W}_{\mathrm{PI}}(n)$ can be used as the initial weight of CMA, $\mathbf{W}_{\mathrm{CMA}}^{(0)}(n)$, in Equation (5). It can enhance interference suppression performance of PI even when the power difference between desired and interference signals is small. Once the desired signal is captured by PI, residual interference signal can be further suppressed in the iteration process of CMA [19]. This approach can be classified as one variant of beamspace CMA (BSCMA) [21,22]. We define this scheme as PI-CMA.

\section{Prototype Specification}

Figure 3 shows the external of the prototype. Table 2 lists the prototype specifications. It is composed of two field programmable gate array (FPGA) boards. Radio frequency (RF) part, modem part, STPA and BAA parts are implemented on one FPGA board while the FEC part is implemented on the other board. The transmitter and receiver are mounted on a single prototype. The receiver has two input ports capable of BAA signal processing. The prototype can also output the spectrum of the received signal after BAA processing as well as the constellations of the specific subcarrier. 


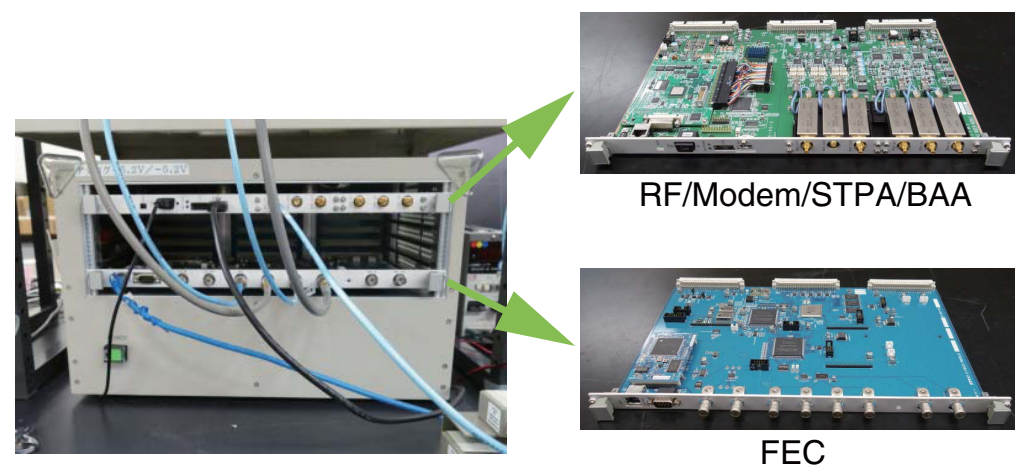

Figure 3. External of the prototype. RF: radio frequency; FEC: forward error correction.

Table 2. Prototype specifications. FPGA: field programmable gate array; FFT: fast Fourier transform; IF: intermediate frequency; D/A: digital-to-analog; A/D: analog-to-digital; BAA: blind adaptive array; CMA: constant modulus algorithm; PI: power inversion.

\begin{tabular}{cc}
\hline Parameters & Values \\
\hline FPGA & Xilinx Vertex-4 \\
Data bus bit-width & $8 \mathrm{bits}$ \\
Clock frequency & $409.6 \mathrm{MHz}$ \\
Sampling rate & $102.4 \mathrm{MHz}$ \\
Number of FFT point & 8192 \\
Frequency resolution & $12.5 \mathrm{kHz}$ \\
Symbol rate per subcarrier & $50 \mathrm{kHz}$ \\
Subcarrier spacing & $100 \mathrm{kHz}$ \\
Number of subcarrier, $N$ & 52 \\
Pulse shaping filter & Root raised cosine, Roll-off factor: 0.2 \\
IF frequency & $140 \mathrm{MHz}$ \\
Resolution of D/A Converter & $16 \mathrm{bits}$ \\
Resolution of A/D Converter & $12 \mathrm{bits}$ \\
Receiver dynamic range & $82 \mathrm{~dB}$ \\
BAA algorithm & $\mathrm{CMA} / \mathrm{PI} / \mathrm{PI}-\mathrm{CMA}$ \\
\hline
\end{tabular}

We employed a fast Fourier transform (FFT) filter bank based multicarrier transmission system. In our first proposal in [19], FFT windowing and BAA per subcarrier were performed on the premise of ideal symbol timing extraction. In the presence of strong inter-system interference, the timing extraction may fail and hence FFT and BAA should be applied before that. In the FFT filter bank, we also utilized an overlapped FFT [23] so as to treat transmission/reception signals as continuous and we can obtain frequency domain signal in arbitrary timing at the receiver side. Filter bank multicarrier system can also keep orthogonality between subcarriers after the FFT time domain windowing function by providing guard bands. Although spectral efficiency is degraded compared to OFDM, the main contribution of this paper is to verify the effectiveness of the proposed STPA-BAA scheme. With this configuration, BAA is applied just after the FFT filter bank, and then followed by original reception process i.e., symbol timing extraction, demodulation, and FEC decoding.

Figure 4 shows a block diagram of the transmitter with STPA. The relationship between the system bandwidth, subcarrier bandwidth, and their arrangement is also depicted. The system bandwidth of the prototype is $102.4 \mathrm{MHz}$ wherein central $5.2 \mathrm{MHz}(=100 \mathrm{kHz} \times 52$ subcarriers) are utilized for signal transmission. Multicarrier transmission signal is firstly generated in the frequency domain. The information bit sequence is encoded by a convolutional encoder. The encoded bits are converted to complex symbols according to the modulation level specified. The symbol rate of each subcarrier is $50 \mathrm{kHz}$ wherein the minimum frequency resolution of the prototype system is $12.5 \mathrm{kHz}$; one symbol is represented by four sample points. Through a serial-to-parallel (S/P) converter, the symbols are mapped to each subcarrier with being shaped to the root raised cosine (RRC) frequency 
response. Roll-off factor of the RRC filter is 0.2 and subcarrier becomes $80 \mathrm{kHz}$ bandwidth. Fifty-two subcarriers are arranged with $100 \mathrm{kHz}$ spacing (i.e., eight points) without inter-carrier interference. In the STPA part, the variable digital attenuator reduces the power of low-level subcarriers to $G \mathrm{~dB}$. In the experiment, the total output power of all subcarriers are increased by an amplifier. Transmission signals for 52 subcarriers are superposed through an 8192 point inverse FFT (IFFT) and then the overlap-add method [24] is applied to obtain the time domain continuous waveform. The multicarrier signals are transmitted after being up-converted to the intermediate frequency (IF). Resolution of the digital-to-analog (D/A) converter is 16 bits.

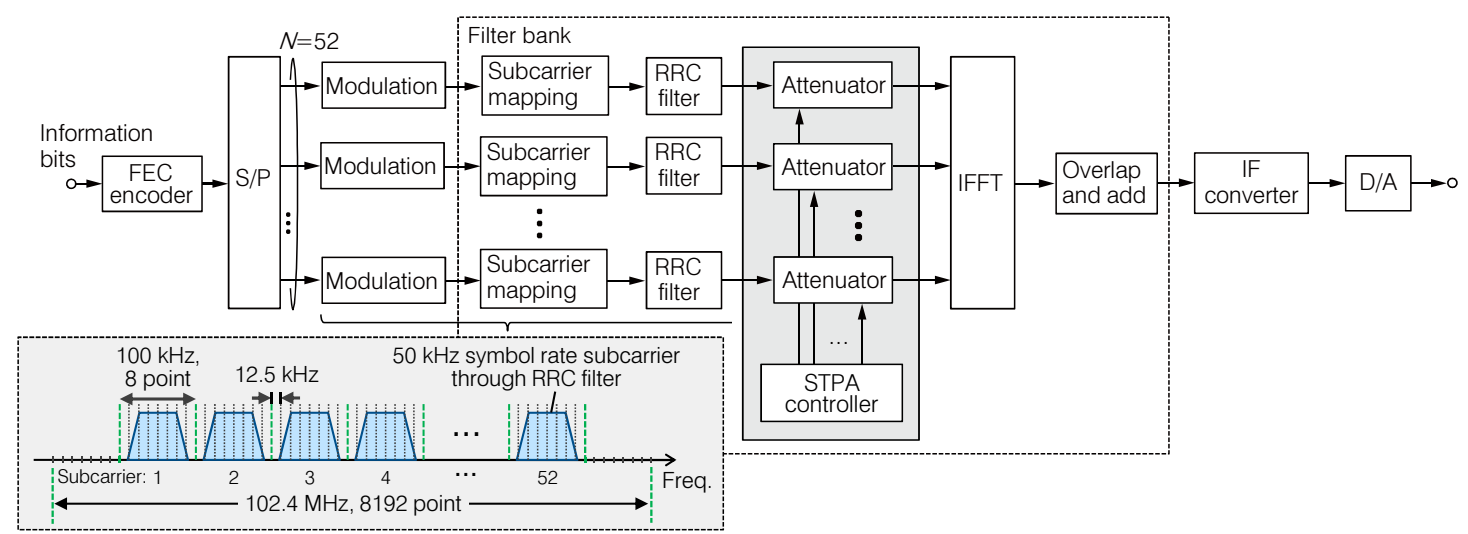

Figure 4. Transmitter employing STPA. S/P: serial-to-parallel; RRC: root raised cosine; IFFT: inverse fast Fourier transform; IF: intermediate frequency; D/A: digital-to-analog.

Figure 5 then shows a block diagram of the receiver with BAA. Dynamic range of the receiver is $82 \mathrm{~dB}$ and resolution of the analog-to-digital (A/D) converter is 12 bits. Each receiver port extracts the signals received on each subcarrier through the overlapped FFT. Extracted subcarriers are individually RRC filtered in the frequency domain and converted to the time domain symbols via 8192 point IFFTs. Each symbol is downsampled to the $50 \mathrm{kHz}$ symbol rate. It is also composed of four sample points. BAA weight for each subcarrier is calculated using the downsampled (but oversampled) symbols according to the pre-assigned subcarrier level (i.e., high or low), and then multiplied to the received signals. CMA (for high-level subcarriers) and PI-CMA (for low-level subcarriers) are implemented as BAA algorithms. After BAA is applied, symbol timing is detected using the unique word (UW) after carrier frequency recovery using a Costas loop [25]. It is performed per subcarrier and the prototype receiver utilizes a successfully estimated one. Finally, decimated symbols are demodulated and decoded to information bits as output after parallel-to-serial (P/S) conversion.

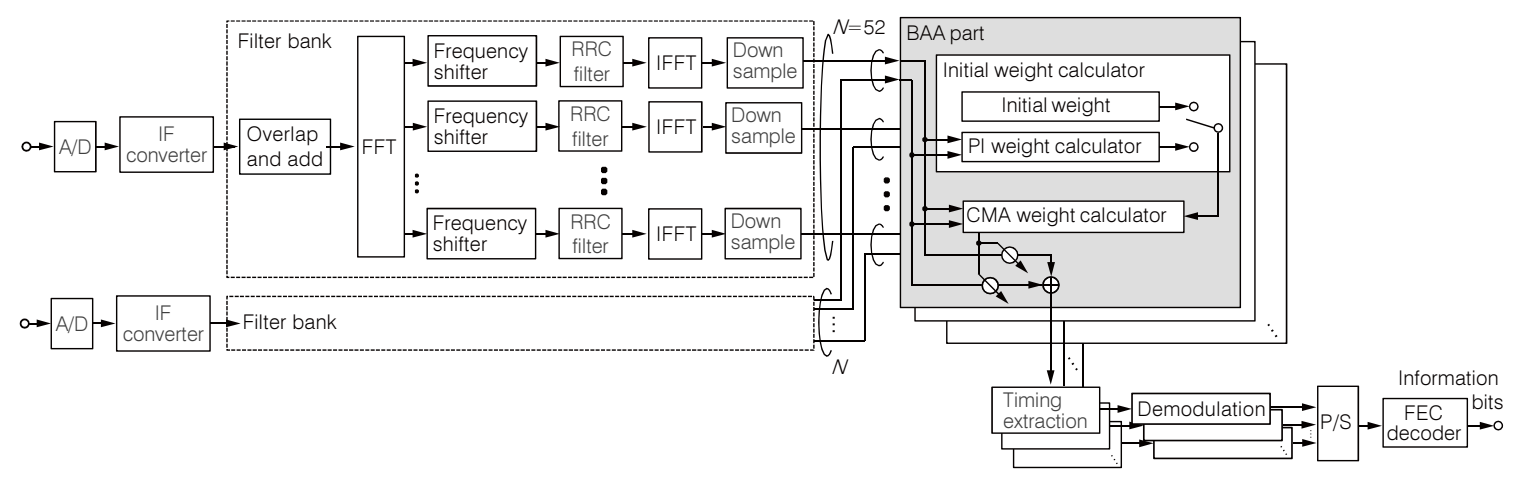

Figure 5. Receiver employing BAA. A/D: analog-to-digital; P/S: parallel-to-serial. 


\section{Laboratory Experiment}

\subsection{Experiment Setup}

Figure 6 shows the wired setup of laboratory experiment and parameters are listed in Table 3. We assumed spectrum superposing scenarios between the primary system and the secondary system with STPA-BAA. Two prototype systems transmit multicarrier signals and interfere with each other. Each signal output from the transmitter is divided and superposed to another system through a variable attenuator (ATT) and a phase shifter. Interference signal level is controlled by the variable attenuator. The phase shifter provides the angle of arrival (AoA) difference between the primary and secondary receivers. AoA difference is set to 20 degrees where desired and interference signals are isolated enough and BAA can effectively suppress interference signal. Additive white Gaussian noise (AWGN) is also added; the signal-to-noise power ratio (SNR) is set to $30 \mathrm{~dB}$. We assumed an interference limited scenario in order to clearly evaluate interference suppression performance of BAA. SNR and SIR are determined by measuring each signal power (desired signal, interference, and noise) just before the receiver input. This wired setup brings a frequency flat channel. Although the practical effectiveness of the STPA-BAA should be evaluated in multipath fading channel, this experiment first verifies its performance from the implementation viewpoint, the quantization levels or the receiver dynamic range.

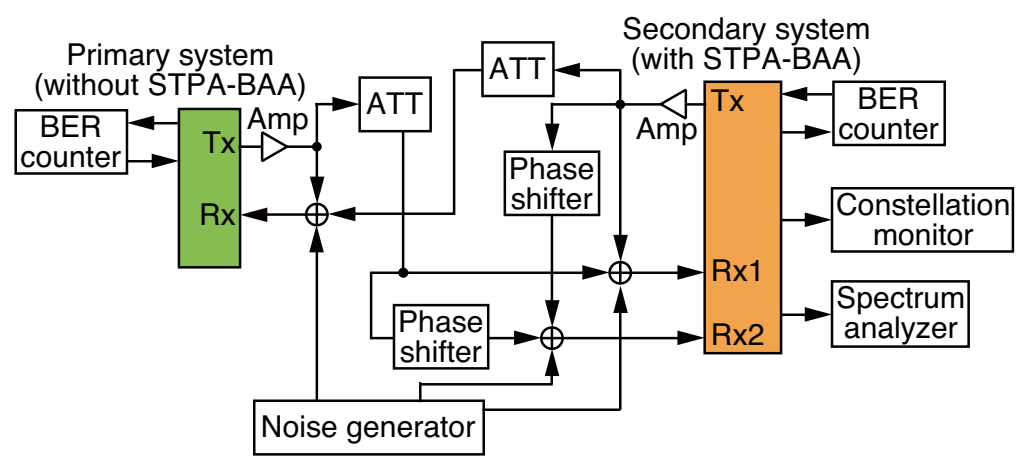

Figure 6. Experiment block diagram. BER: bit error rate; ATT: attenuator.

Table 3. Experiment parameters.

\begin{tabular}{cc}
\hline Parameters & Values \\
\hline Number of Transmission antenna & 1 (Primary system) \\
Number of Reception antenna & 1 (Secondary system) \\
Modulation scheme & 1 (Primary system) \\
FEC (forward error correction) scheme & 2 (Secondary system) \\
Constraint length & QPSK (quadrature phase shift keying) \\
FEC Coding rate & Convolutional code \\
FEC Decoder & 7 \\
Bit interleave & $1 / 2$ \\
Channel & Viterbi \\
None \\
Angle of arrival difference & Frequency flat \\
Number of sample for BAA, $S$ & $30 \mathrm{~dB}$ \\
CMA iteration number & $20^{\circ}$ \\
High/Low-level subcarrier power ratio, $\mathrm{G}$ & $512(128$ symbols) \\
Number of high-level subcarrier, $N_{\text {High }}$ & 10 \\
\hline
\end{tabular}




\subsection{Experiment Results of the Secondary System}

Figure 7 shows the in-phase and quadrature (IQ) constellation upon the application of BAA with/without STPA at $\mathrm{SIR}_{\text {total }}=0 \mathrm{~dB}, G=20 \mathrm{~dB}$, and $N_{\text {High }}=7$. In BAA without STPA, the IQ constellation of the received signal is spread due to the unsuppressed interference; the resulting bit error rate (BER) was 0.5. BAA with STPA can effectively suppress the interference and thus the IQ constellation converged for both high and low-level subcarriers. Meanwhile, the amplitude of the IQ constellation at the low-level subcarriers was smaller than that at high-level subcarriers. It is obvious but enlarging the value of $G$ degrades the reception performance of the low-level subcarriers.

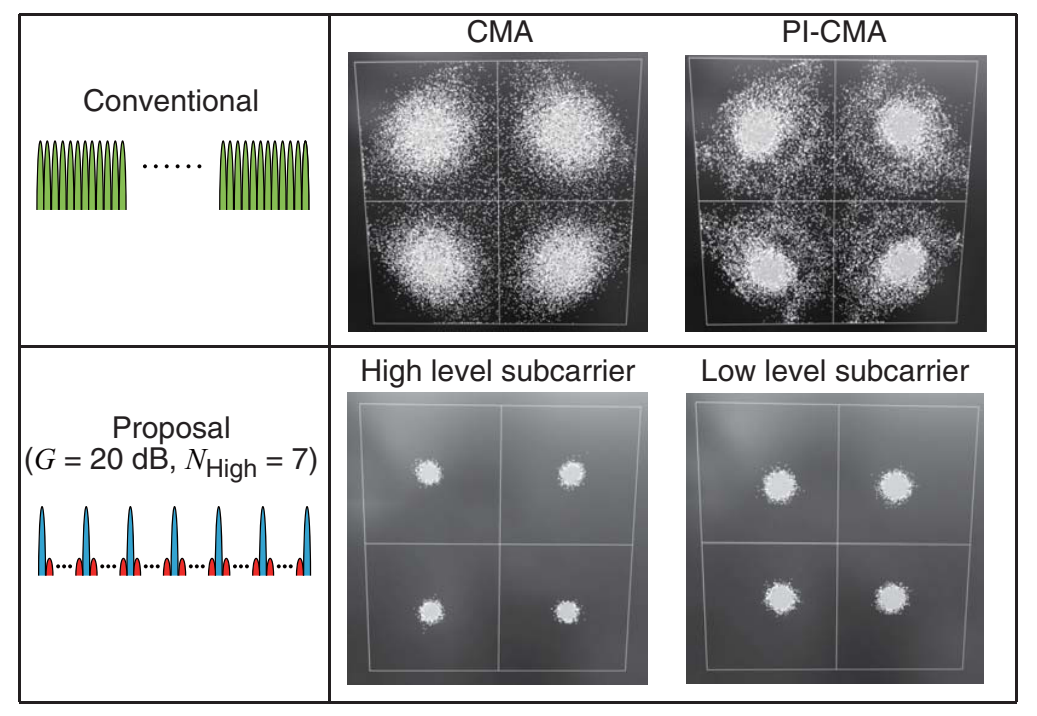

Figure 7. In-phase and quadrature (IQ) constellation by BAA at $\operatorname{SIR}_{\text {total }}=0 \mathrm{~dB}(\mathrm{G}=20 \mathrm{~dB}$ and $N_{\text {High }}=7$ ).

Figure 8a shows the BER performance as a function of $\operatorname{SIR}_{\text {total }}$ of the secondary system with a comparison to the simulation results. This figure shows the fundamental BER performance in the case that CMA, PI and PI-CMA are applied to all subcarriers without STPA. Simulation was conducted in the equivalent baseband domain assuming ideal conditions, without quantization nor oversampling. The $\mathrm{SIR}_{\text {total }}$ offset of the experiment results to the simulated ones is within $2 \mathrm{~dB}$ except for the original PI. Since the BAA is applied to oversampled signals whose envelope is not constant, interference suppression ability is slightly weakened. In addition to that, discrepancy between two results of PI becomes larger than that of CMA and PI-CMA. It is because of excessive interference signal level to the receiver inputs. In the lower $\mathrm{SIR}_{\text {total }}$ region, large power of the interference signal is input and the desired signal power is accordingly reduced so as not to exceed the dynamic range of the receiver inputs. It thus causes bit resolution shortage for the desired signal and the interference suppression performance of PI to deteriorate. Meanwhile, it can be confirmed that PI-CMA exhibits improved BER performance compared to the original PI. CMA utilizing PI as an initial weight can greatly enhance interference suppression performance. The legacy CMA and PI-CMA cannot suppress interference signal when $\operatorname{SIR}_{\text {total }}$ is around $0 \mathrm{~dB}$.

Figure $8 \mathrm{~b}$ shows the BER performance of the secondary system with STPA-BAA with parameters $G=20 \mathrm{~dB}$ and $N_{\mathrm{High}}=3$ and 7 . The proposed scheme effectively works in the region around $\mathrm{SIR}_{\text {total }}=0 \mathrm{~dB}$ and improves the BER performance. Here, we define the operational region of the secondary system as the range to which $\operatorname{SIR}_{\text {total }}$ holds the BER under $10^{-5}$. In the case of Figure $8 \mathrm{~b}$, the upper and lower bounds that satisfy requirements are 11 and $-9 \mathrm{~dB}$, respectively; operational $\operatorname{SIR}_{\text {total }}$ range is thus derived as $20 \mathrm{~dB}$. Focusing on the upper bound, BER behavior of the experiment matches that of the simulation well and they exhibit almost the same upper bound $\operatorname{SIR}_{\text {total }}$ value. In the higher $\operatorname{SIR}_{\text {total }}$ region, interference signal level is slight, so that the sufficient 
dynamic range can be kept wherein BAAs suppress interference well. Although BER behaviors of the experiment and simulation results are different especially in the lower SIR $_{\text {total }}$ region, they almost coincide and satisfactory operational $\operatorname{SIR}_{\text {total }}$ range can be ensured.

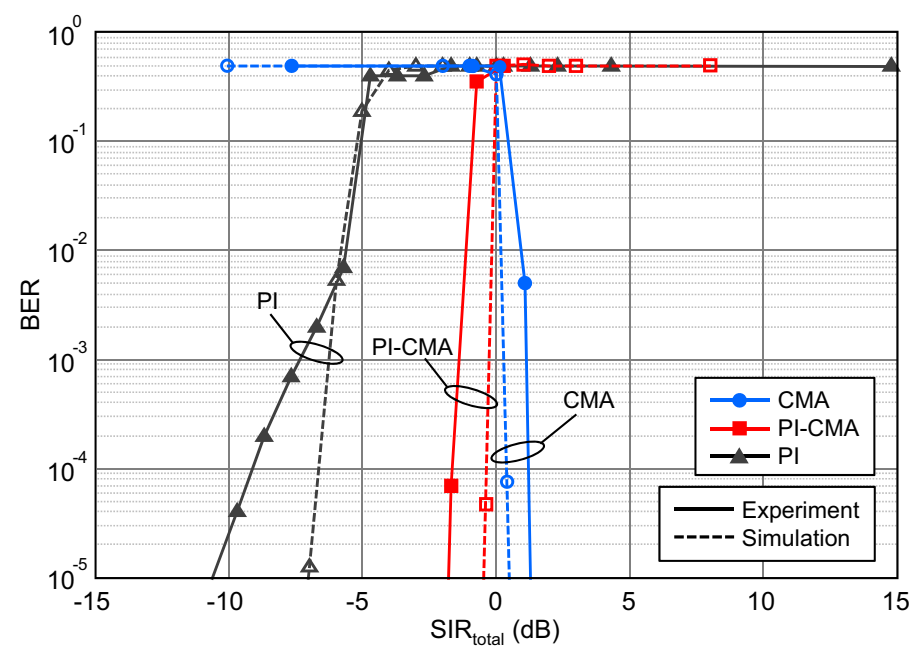

(a) Without STPA

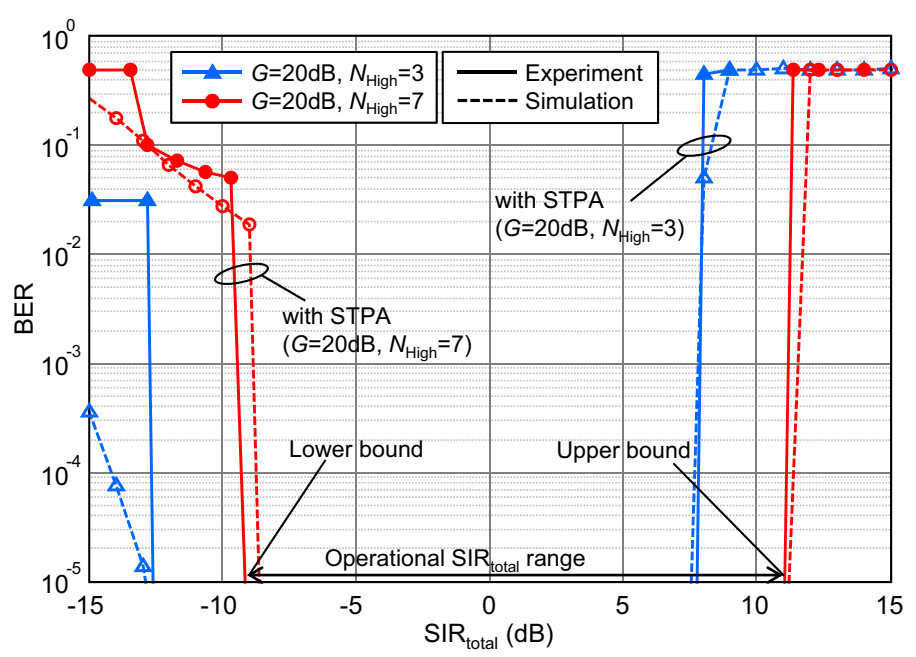

(b) With STPA

Figure 8. BER performance of the secondary system. (a) without STPA; (b) with STPA. STPA: subcarrier transmission power assignment.

The operational $\operatorname{SIR}_{\text {total }}$ range of the secondary system is revealed by the subcarrier power ratio, $G$, and the high-level subcarrier number, $N_{\text {High }}$. Figure 9 shows the upper and lower bounds of the operational $\operatorname{SIR}_{\text {total }}$ as a parameter of $G$ when $N_{\text {High }}$ is set to 7 . This experiment firstly measured the BER characteristics at the specified parameter of $G$ and then obtained the upper and lower bounds as indicated in Figure $8 \mathrm{~b}$. The operational $\mathrm{SIR}_{\text {total }}$ range can be seen as the difference between upper and lower bounds. For comparison, the computer simulation results are also plotted. Basically, operational $\operatorname{SIR}_{\text {total }}$ range exhibits the same value as $G$ because of the wired and flat fading channel. Experiment results show almost comparable performance to the simulation one. However, non-negligible deviation can be observed at $G=24 \mathrm{~dB}$, and we confirmed that the prototype does not work at $G=26 \mathrm{~dB}$. The conceivable reason is quantization resolution shortage of low-level subcarriers. STPA function of the prototype controls the power density of low-level subcarrier by digital attenuator, i.e., reducing the number of digits. Furthermore, $24 \mathrm{~dB}$ power attenuation is done 
by discarding the rightmost 8 bits; $10 \log _{10}\left(2^{8}\right)=24.08 \mathrm{~dB}$. At the receiver side, resolution for $24 \mathrm{~dB}$ attenuated symbols of low-level subcarrier is only $4(=12-8)$ bits or less. It would degrade calculation accuracy of PI-CMA weight and thus interference cannot be well suppressed.

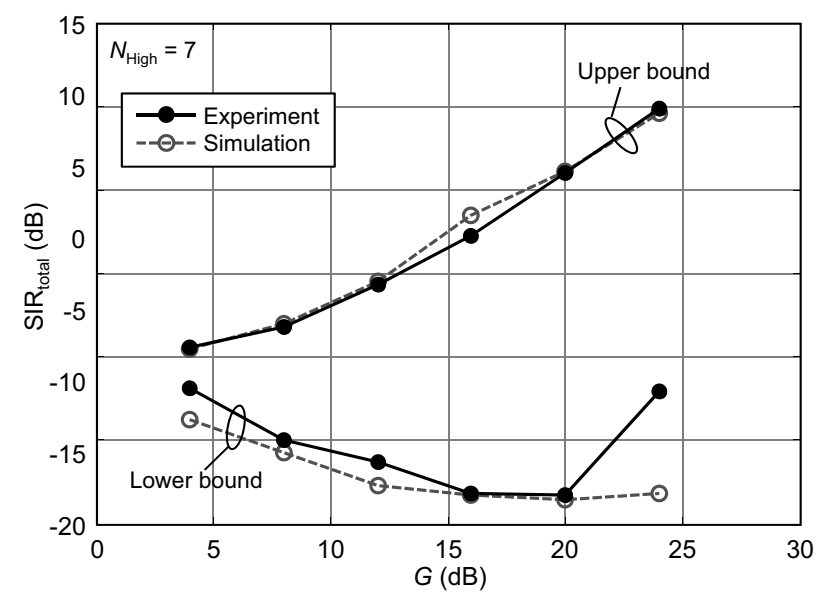

Figure 9. Operational $\operatorname{SIR}_{\text {total }}$ range of the secondary system by subcarrier power ratio $G\left(N_{\text {High }}=7\right)$.

Figure 10 shows the upper and lower bounds of the operational $\operatorname{SIR}_{\text {total }}$ as a function of $N_{\text {High }}$. When $N_{\text {High }}$ decreases, the surplus power is reassigned to all subcarriers and hence the power density of overall subcarriers are increased. Accordingly, the power density of the interference signal to be suppressed by the secondary system is increased and the operational SIR total range is shifted to the lower side as $N_{\text {High }}$ is decreased. Here, operational SIR $_{\text {total }}$ in the lower bound by experiment shrinks at $N_{\text {High }} \leq 2$ compared to the simulation results. As mentioned above, it is due to the bit resolution shortage caused by ensuring the dynamic range of receiver inputs for larger interference signal power. This impact becomes more significant especially in low-level subcarriers. Increasing the number of quantization bits or enlarging dynamic range could avoid such degradations. These parameters should be optimized depending on the secondary system design.

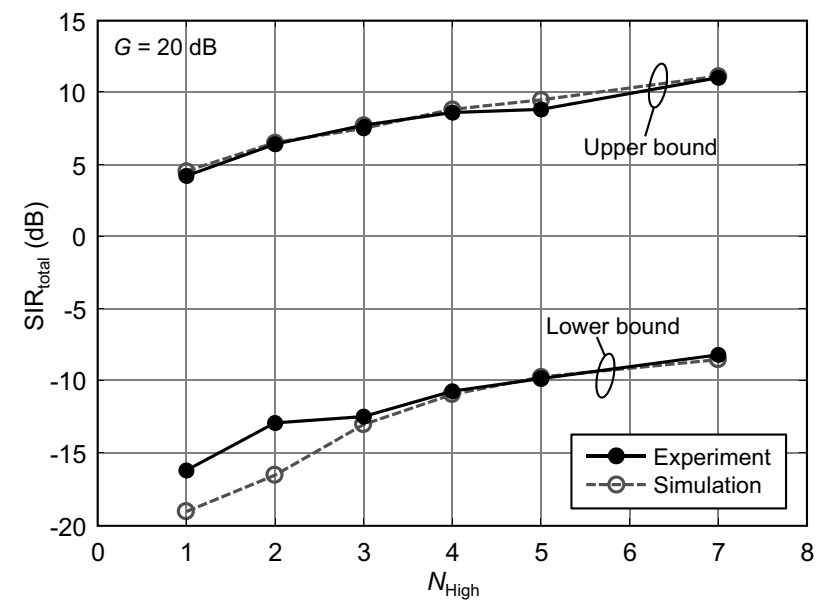

Figure 10. Operational $\operatorname{SIR}_{\text {total }}$ range of the secondary system by number of high level subcarrier $N_{\text {High }}$ $(G=20 \mathrm{~dB})$.

\subsection{Experiment Results of the Primary System}

Finally, we reveal the operational SIR $_{\text {total }}$ improvement of the primary system by STPA. Figure 11 shows the BER performances of experiment and simulation as a function of SIR at the primary system. The parameters of the secondary system are $G=20$ and $24 \mathrm{~dB}$, and $N_{\mathrm{High}}=1,3,4,7$. 
For comparison, BER performance is depicted when the secondary system does not perform STPA. When $N_{\text {High }}$ is 3 or lower (see Figure 11a), BER performance of the primary system can be improved. It should be noted that performance of the primary system depends on the symbol timing accuracy. Since the interference power within the bandwidth cannot be changed, the primary receiver should precisely detect the symbol timing in the interference dominant situation. As described in Section 3 , the prototype receiver utilizes the symbol timing of a successfully estimated subcarrier. If inter-system interference from the secondary system is reduced on certain subcarriers, the primary receiver can extract symbol timing for demodulation and thus BER performance is improved. Meanwhile, when $N_{\mathrm{High}}$ is larger than 3 (see Figure 11b), BER performance of the experiment becomes worse than that of the simulation. The large number of high-level subcarriers might affect over all BER characteristics of the primary receiver. Although FEC function is expected to compensate its undesired degradation, detailed mechanisms should be further investigated. SIR total improvements at BER $=10^{-5}$ in experiments are $2.5 \mathrm{~dB}$ at $\left(G, N_{\text {High }}\right)=(24 \mathrm{~dB}, 1), 3.0 \mathrm{~dB}$ at $\left(G, N_{\text {High }}\right)=(20 \mathrm{~dB}, 3)$ and $7.0 \mathrm{~dB}$ at $\left(G, N_{\text {High }}\right)=(24 \mathrm{~dB}, 3)$, respectively. Allowing degradation of interference suppression performance for the proposed scheme, larger value of $G$ and lower value of $N_{\text {High }}$ can provide more effective interference reduction for the primary system. In addition, the experiment results match the computer simulation ones well except for the primary system with $N_{\text {High }}>3$.

The above experiments verified a fundamental feasibility of the proposed spectrum superposing approach via STPA-BAA using a wired setup. For practical applicability, experiments should be conducted in outdoor environments where multipath components exist. Multipath fading might dynamically fluctuate levels of high and low subcarriers and it weakens interference suppression performance of BAAs. Although our simulative work showed the effectiveness of the proposal [19], more practical performance should be further investigated. In this case, OFDM is preferable since cyclic prefix (CP) can mitigate the inter-symbol interference (ISI) caused by the multipath effect. Here, challenges arise regarding how to extract an exact timing for FFT windowing in interference limited situations. FFT windowing should be performed before BAA processing per subcarrier; therefore, the timing extraction should be done in the presence of large inter-system interference, which causes the detection accuracy to deteriorate. Resolving the above issues, its overall effectiveness will be disclosed under practical application scenarios, e.g., LAA or LTE-U.

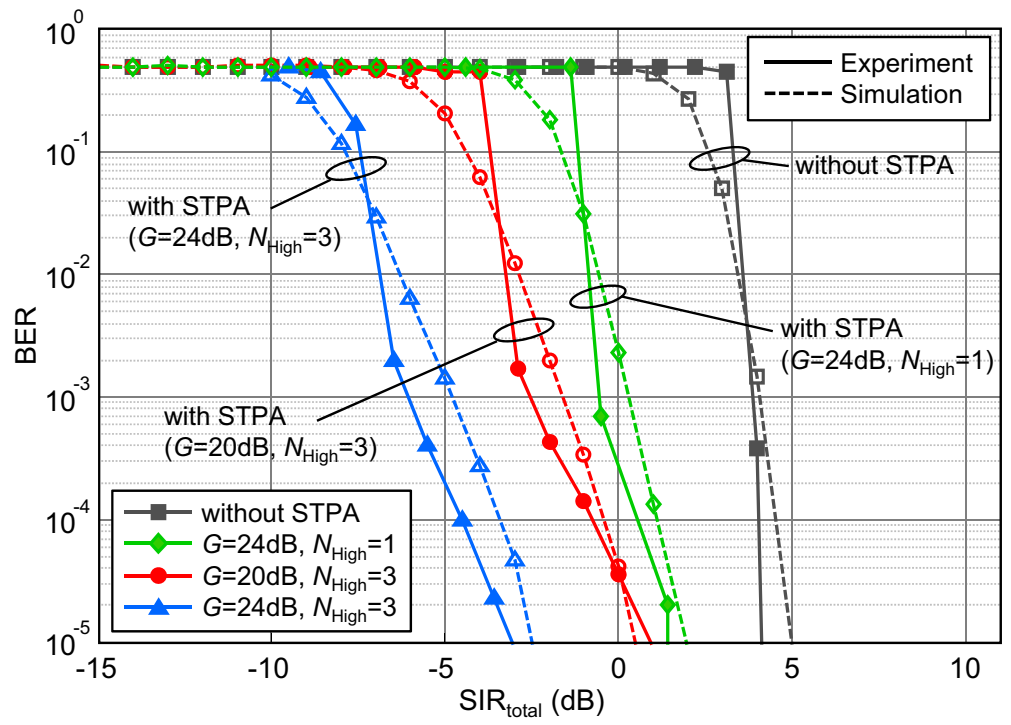

(a) $N_{\text {High }} \leq 3$

Figure 11. Cont. 


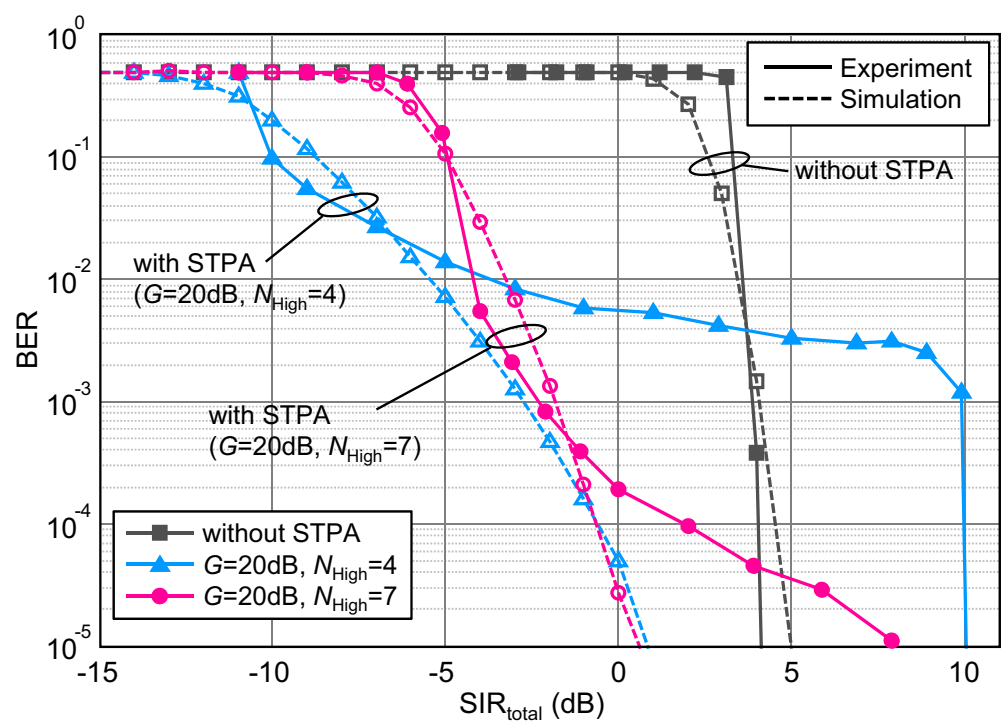

(b) $N_{\text {High }}>3$

Figure 11. BER performance of the primary system. (a) $N_{\text {High }} \leq 3 ;$ (b) $N_{\text {High }}>3$.

\section{Conclusions}

This paper experimentally verified the fundamental feasibility of our spectrum superposing scheme enabled by the proposed STPA-BAA. A prototype was fabricated and its performance in two multicarrier transmission systems was evaluated through a wired setup. The experiment demonstrated that STPA-BAA can suppress interference from the primary transmitter at $\operatorname{SIR}_{\text {total }}$ of around $0 \mathrm{~dB}$ and can reduce interference to the primary receiver. Its practical applicable regions have been disclosed under some limitations in interference suppression ability due to the quantization or dynamic range of the transceiver. The most significant feature of the proposed scheme is that there is no need to modify the existing primary system. More detailed feasibility of the STPA-BAA scheme will be further investigated in more practical environments such as frequency selective fading channels.

Acknowledgments: The authors are sincerely grateful to Yutaka Sakuda, Michiaki Nagai, and Hiroshi Morooka of NTT Advanced Technology Corporation for their support on hardware implementation and prototype fabrication. The authors also give their sincere thanks to Takatoshi Sugiyama of Kogakuin University and Jun Mashino of NTT Docomo Inc. for their coordination of this work.

Author Contributions: H.S. and K.S. conducted experiments and analyzed the data; K.M. conceived the proposed scheme, provided simulation results and directed prototype fabrication; and H.S. and K.M. wrote the paper.

Conflicts of Interest: The authors declare no conflicts of interest.

\section{Abbreviations}

The following abbreviations are used in this manuscript:

$\begin{array}{ll}\text { Wi-Fi } & \text { Wireless Fidelity } \\ \text { WiMAX } & \text { World interoperability for Microwave Access } \\ \text { LTE } & \text { Long Term Evolution } \\ \text { OSA } & \text { Opportunistic Spectrum Access } \\ \text { DSA } & \text { Dunamic Spectrum Access } \\ \text { LTE-U } & \text { LTE in Unlicensed spectrum } \\ \text { LAA } & \text { Licensed Assisted Access } \\ \text { LBT } & \text { Listen Before Talk } \\ \text { RTS } & \text { Request-to-Send } \\ \text { CTS } & \text { Clear-to-Send } \\ \text { OFDM } & \text { Orthogonal Frequency Division Multiplexing }\end{array}$




$\begin{array}{ll}\text { CP } & \text { Cyclic Prefix } \\ \text { ISI } & \text { Inter-Symbol Interference } \\ \text { FEC } & \text { Forward Error Correction } \\ \text { MIMO } & \text { Multiple-Input Multiple-Output } \\ \text { STPA } & \text { Subcarrier Transmission Power Assignment } \\ \text { BAA } & \text { Blind Adaptive Array } \\ \text { CMA } & \text { Constant Modulus Algorithm } \\ \text { PI } & \text { Power Inversion } \\ \text { FPGA } & \text { Field Programmable Gate Array } \\ \text { RF } & \text { Radio Frequency } \\ \text { IF } & \text { Intermediate Frequency } \\ \text { S/P } & \text { Serial-to-Parallel } \\ \text { P/S } & \text { Parallel-to-Serial } \\ \text { D/A } & \text { Digital-to-Analog } \\ \text { A/D } & \text { Analog-to-Digital } \\ \text { RRC } & \text { Root Raised Cosine } \\ \text { FFT } & \text { Fast Fourier Transform } \\ \text { IFFT } & \text { Inverse Fast Fourier Transform } \\ \text { UW } & \text { Unique Word } \\ \text { AoA } & \text { Angle of Arrival } \\ \text { ATT } & \text { Attenuator } \\ \text { AWGN } & \text { Additive white Gaussian noise } \\ \text { QPSK } & \text { Quadrature Phase Shift Keying } \\ \text { SNR } & \text { Signal-to-Noise power Ratio } \\ \text { SIR } & \text { Signal-to-Interference power Ratio } \\ \text { BER } & \text { Bit Error Rate } \\ & \end{array}$

\section{References}

1. Boccardi, F.; Heath, R.W.; Lozano, A.; Marzetta, T.L.; Popovski, P. Five disruptive technology directions for 5G. IEEE Commun. Mag. 2014, 52, 74-80.

2. Spencer, Q.H.; Swindlehurst, A.L.; Haardt, M. Zero-forcing methods for downlink spatial multiplexing in multiuser MIMO channels. IEEE Trans. Signal Process. 2004, 52, 461-471.

3. Haykin, S. Cognitive radio: Brain-empowered wireless communications. IEEE J. Sel. Areas Commun. 2005, 23, 201-220.

4. Zhao, Q. Spectrum Opportunity and Interference Constraint in Opportunistic Spectrum Access. In Proceedings of the 2007 IEEE International Conference on Acoustics, Speech and Signal Processing-ICASSP, Honolulu, HI, USA, 16-20 April 2007; Volume 3, pp. III-605-III-608.

5. Zhao, Q.; Sadler, B.M. A Survey of Dynamic Spectrum Access. IEEE Signal Process. Mag. 2007, 24, 79-89.

6. Chang, G.Y.; Huang, J.F. A Fast Rendezvous Channel-Hopping Algorithm for Cognitive Radio Networks. IEEE Commun. Lett. 2013, 17, 1475-1478.

7. Takyu, O.; Yamakita, T.; Fujii, T.; Ohta, M.; Sasamori, F.; Handa, S. Optimization of Learning Time for Learning-Assisted Rendezvous Channel in Cognitive Radio System. IEICE Trans. Commun. 2015, 98, 360-369.

8. Valls, V.; Garcia-Saavedra, A.; Costa, X.; Leith, D.J. Maximizing LTE Capacity in Unlicensed Bands (LTE-U/LAA) While Fairly Coexisting With 802.11 WLANs. IEEE Commun. Lett. 2016, 20, 1219-1222.

9. Jian, Y.; Shih, C.F.; Krishnaswamy, B.; Sivakumar, R. Coexistence of Wi-Fi and LAA-LTE: Experimental evaluation, analysis and insights. In Proceedings of the 2015 IEEE International Conference on Communication Workshop (ICCW), London, UK, 8-12 June 2015; pp. 2325-2331.

10. Song, Y.; Sung, K.W.; Han, Y. Coexistence of Wi-Fi and Cellular With Listen-Before-Talk in Unlicensed Spectrum. IEEE Commun. Lett. 2016, 20, 161-164.

11. Nishimori, K.; Taranto, R.D.; Yomo, H.; Popovski, P.; Takatori, Y.; Prasad, R.; Kubota, S. Spatial Opportunity for Cognitive Radio Systems with Heterogeneous Path Loss Conditions. In Proceedings of the 2007 IEEE 65th Vehicular Technology Conference-VTC2007-Spring, Dublin, Ireland, 22-25 April 2007; pp. 2631-2635. 
12. Park, J.; Park, Y.; Hwang, S.; Jeong, B.J. Low-Complexity GSVD-Based Beamforming and Power Allocation for a Cognitive Radio Network. IEICE Trans. Commun. 2012, 95, 3536-3544.

13. Sodagari, S. On effects of imperfect channel state information on null space based cognitive MIMO communication. In Proceedings of the 2015 International Conference on Computing, Networking and Communications (ICNC), Anaheim, CA, USA, 16-19 February 2015; pp. 438-444.

14. Treichler, J.; Agee, B. A new approach to multipath correction of constant modulus signals. IEEE Trans. Acoust. Speech Signal Process. 1983, 31, 459-472.

15. Agee, B. The least-squares CMA: A new technique for rapid correction of constant modulus signals. In Proceedings of the IEEE International Conference on Acoustics, Speech, and Signal Processing (ICASSP), Tokyo, Japan, 7-11 April 1986; Volume 11, pp. 953-956.

16. Compton, R.T. The Power-Inversion Adaptive Array: Concept and Performance. IEEE Trans. Aerosp. Electron. Syst. 1979, AES-15, 803-814.

17. Ukawa, T.; Fujimoto, M.; Hori, T. Optimal snapshot number of power inversion adaptive array in ITS communication environment. In Proceedings of the 2013 Asia-Pacific Microwave Conference Proceedings (APMC), Seoul, Korea, 5-8 November 2013; pp. 563-565.

18. Maruta, K.; Mashino, J.; Sugiyama, T. Blind adaptive arrays with subcarrier transmission power assignment for spectrum superposing. In Proceedings of the 2014 Asia-Pacific Microwave Conference, Sendai, Japan, 4-7 November 2014; pp. 567-569.

19. Maruta, K.; Mashino, J.; Sugiyama, T. Blind Interference Suppression Scheme by Eigenvector Beamspace CMA Adaptive Array with Subcarrier Transmission Power Assignment for Spectrum Superposing. IEICE Trans. Commun. 2015, 98, 1050-1057.

20. So, H.; Maruta, K.; Mashino, J.; Suzaki, K. Experimental Verification of Spectrum Superposing in Two Different Systems by Blind Adaptive Array with Subcarrier Transmission Power Assignment. In Proceedings of the 2016 IEEE 84th Vehicular Technology Conference (VTC-Fall), Montreal, QC, Canada, 18-21 September 2016; pp. 1-5.

21. Chiba, I.; Chujo, W.; Fujise, M. Beam space constant modulus algorithm adaptive array antennas. In Proceedings of the 1993 Eighth International Conference on Antennas and Propagation, Edinburgh, UK, 30 March-2 April 1993; Volume 2, pp. 975-978.

22. Nishimori, K.; Kikuma, N.; Inagaki, N. The Differential CMA Adaptive Array Antenna Using an Eigen-Beamspace System. IEICE Trans. Commun. 1995, 78, 1480-1488.

23. Tanabe, M.; Umehira, M. Design and Performance of Overlap FFT Filter-Bank for Dynamic Spectrum Access Applications. IEICE Trans. Commun. 2012, 95, 1249-1255.

24. Oppenheim, A.V.; Schafer, R.W.; Buck, J.R. Discrete-Time Signal Processing, 2nd ed.; Prentice-Hall, Inc.: Upper Saddle River, NJ, USA, 1999.

25. Costas, J. Synchronous Communications. IRE Trans. Commun. Syst. 1957, 5, 99-105. 